\title{
REFINEMENTS OF A REVERSED AM-GM OPERATOR INEQUALITY
}

\author{
MOJTABA BAKHERAD
}

\begin{abstract}
We prove some refinements of a reverse AM-GM operator inequality due to M. Lin [Studia Math. 2013;215:187-194]. In particular, we show the operator inequality

$$
\Phi^{p}\left(A \nabla_{\nu} B+2 r M m\left(A^{-1} \nabla B^{-1}-A^{-1} \sharp B^{-1}\right)\right) \leq \alpha^{p} \Phi^{p}\left(A \sharp_{\nu} B\right),
$$

where $A, B$ are positive operators on a Hilbert space such that $0<m \leq A, B \leq M$ for some positive numbers $m, M, \Phi$ is a positive unital linear map, $\nu \in[0,1]$, $r=\min \{\nu, 1-\nu\}, p>0$ and $\alpha=\max \left\{\frac{(M+m)^{2}}{4 M m}, \frac{(M+m)^{2}}{4^{\frac{2}{p}} M m}\right\}$.
\end{abstract}

\section{INTRODUCTION AND PRELIMINARIES}

Let $\mathbb{B}(\mathscr{H})$ denote the $C^{*}$-algebra of all bounded linear operators on a complex Hilbert space $\mathscr{H}$, with the identity $I$. In the case when $\operatorname{dim} \mathscr{H}=n$, we identify $\mathbb{B}(\mathscr{H})$ with the matrix algebra $\mathbb{M}_{n}$ of all $n \times n$ matrices with entries in the complex field. An operator $A \in \mathbb{B}(\mathscr{H})$ is called positive if $\langle A x, x\rangle \geq 0$ for all $x \in \mathscr{H}$, and we then write $A \geq 0$. We write $A>0$ if $A$ is a positive invertible operator. For self-adjoint operators $A, B \in \mathbb{B}(\mathscr{H})$ we say that $A \leq B$ if $B-A \geq 0$. The Gelfand map $f(t) \mapsto f(A)$ is an isometrical $*$-isomorphism between the $C^{*}$-algebra $C(\operatorname{sp}(A))$ of continuous functions on the spectrum $\operatorname{sp}(A)$ of a self-adjoint operator $A$ and the $C^{*}$-algebra generated by $A$ and $I_{\mathscr{H}}$. If $f, g \in C(\operatorname{sp}(A))$, then $f(t) \geq g(t)(t \in \operatorname{sp}(A))$ implies that $f(A) \geq g(A)$.

Let $A, B \in \mathbb{B}(\mathscr{H})$ be two positive invertible operators and $\nu \in[0,1]$. The operator weighted arithmetic, geometric and harmonic means are defined by $A \nabla_{\nu} B=$ $(1-\nu) A+\nu B, A_{\sharp_{\nu}} B=A^{\frac{1}{2}}\left(A^{\frac{-1}{2}} B A^{\frac{-1}{2}}\right)^{\nu} A^{\frac{1}{2}}$ and $A !_{\nu} B=\left((1-\nu) A^{-1}+\nu B^{-1}\right)^{-1}$, respectively. In particular, for $\nu=\frac{1}{2}$ we get the usual operator arithmetic mean $\nabla$,

2010 Mathematics Subject Classification. Primary 47A63, Secondary 47A60.

Key words and phrases. the operator arithmetic mean, the operator geometric mean, the operator harmonic mean, positive unital linear map, reverse AM-GM operator inequality. 
the geometric mean $\sharp$ and the harmonic mean !. The AM-GM inequality reads

$$
\frac{A+B}{2} \geq A \sharp B,
$$

for all positive operators $A, B$. It is shown in [10] the following reverse of AM-GM inequality involving positive linear maps

$$
\Phi\left(\frac{A+B}{2}\right) \leq \frac{(M+m)^{2}}{4 M m} \Phi(A \sharp B),
$$

where $0<m \leq A, B \leq M$ and $\Phi$ is a positive unital linear map.

For two positive operators $A, B \in \mathbb{B}(\mathscr{H})$, the Löwner-Heinz inequality states that, if $A \leq B$, then

$$
A^{p} \leq B^{p}, \quad(0 \leq p \leq 1)
$$

In general $(1.2)$ is not true for $p>1$. Lin [10, Theorem 2.1] showed however a squaring of (1.1), namely that the inequality

$$
\Phi^{2}\left(\frac{A+B}{2}\right) \leq\left(\frac{(M+m)^{2}}{4 M m}\right)^{2} \Phi^{2}(A \sharp B)
$$

as well as

$$
\Phi^{2}\left(\frac{A+B}{2}\right) \leq\left(\frac{(M+m)^{2}}{4 M m}\right)^{2}(\Phi(A) \sharp \Phi(B))^{2}
$$

hold. Using inequality (1.2) we therefore get

$$
\Phi^{p}\left(\frac{A+B}{2}\right) \leq\left(\frac{(M+m)^{2}}{4 M m}\right)^{p} \Phi^{p}(A \sharp B) \quad(0<p \leq 2)
$$

and

$$
\Phi^{p}\left(\frac{A+B}{2}\right) \leq\left(\frac{(M+m)^{2}}{4 M m}\right)^{p}(\Phi(A) \sharp \Phi(B))^{p} \quad(0<p \leq 2),
$$

where $0<m \leq A, B \leq M$ and $\Phi$ is a positive unital linear map.

In [13] the authors extended (1.3) and (1.4) to $p>2$. They proved that the inequalities

$$
\Phi^{p}\left(\frac{A+B}{2}\right) \leq\left(\frac{(M+m)^{2}}{4^{\frac{2}{p}} M m}\right)^{p} \Phi^{p}(A \sharp B) \quad(p>2)
$$

and

$$
\Phi^{p}\left(\frac{A+B}{2}\right) \leq\left(\frac{(M+m)^{2}}{4^{\frac{2}{p}} M m}\right)^{p}(\Phi(A) \sharp \Phi(B))^{p} \quad(p>2),
$$

where $0<m \leq A, B \leq M$. In [4] and [12] the authors showed that

$$
\Phi^{p}(A \sigma B) \leq \alpha^{p} \Phi^{p}(A \tau B),
$$


and

$$
\Phi^{p}(A \sigma B) \leq \alpha^{p}(\Phi(A) \tau \Phi(B))^{p},
$$

where $0<m \leq A, B \leq M, \Phi$ be a positive unital linear map, $\sigma, \tau$ be two arbitrary means between harmonic and arithmetic means, $\alpha=\max \left\{\frac{(M+m)^{2}}{4 M m}, \frac{(M+m)^{2}}{4^{\frac{2}{p}} M m}\right\}$ and $p>0$. Choi's inequality (see e.g. [1, p. 41]) reads

$$
\Phi(A)^{-1} \leq \Phi\left(A^{-1}\right)
$$

for any positive unital linear map $\Phi$ and operator $A>0$. Choi's inequality cannot be squared [10], but a reverse of Choi's inequality (known as the operator Kantorovich inequality) can be squared, see e.g. [11].

In this paper, we present some refinements of inequalities (1.5) and (1.6) under some mild conditions for $0<p \leq 1$ and some refinements of inequalities (1.7) and (1.8) for the operator norm and $p>2$. We also show a refinement of the operator Pólya-Szegö inequality.

\section{MAin RESUlts}

We need the following lemmas to prove our results.

Lemma 2.1. [3] Let $A, B>0$. Then

$$
\|A B\| \leq \frac{1}{4}\|A+B\|^{2} .
$$

Lemma 2.2. [8] Let $A, B \geq 0$ and $p>1$. Then

$$
\left\|A^{p}+B^{p}\right\| \leq\left\|(A+B)^{p}\right\|
$$

Lemma 2.3. Let $A, B>0$ and $\alpha>0$. Then $A \leq \alpha B$ if and only if $\left\|A^{\frac{1}{2}} B^{\frac{-1}{2}}\right\| \leq \alpha^{\frac{1}{2}}$.

Proof. Obviously, $A \leq \alpha B$ if and only if $B^{\frac{-1}{2}} A B^{\frac{-1}{2}} \leq \alpha$. By definition, this holds if and only if $\left\|A^{\frac{1}{2}} B^{\frac{-1}{2}}\right\|^{2} \leq \alpha$ and the proof is complete.

Lemma 2.4. [4] Let $0<m \leq A, B \leq M$, $\Phi$ be a positive unital linear map and $\sigma$, $\tau$ be two arbitrary means between harmonic and arithmetic means. Then

$$
\Phi(A \sigma B)+M m \Phi^{-1}(A \tau B) \leq M+m
$$

In the next proposition we extend the inequalities (1.9) and (1.10) to $p>2$ and the inequalities (1.7) and (1.8) to arbitrary means between harmonic and arithmetic means. 
Proposition 2.5. Let $0<m \leq A, B \leq M, \Phi$ be a positive unital linear map, $\sigma, \tau$ be two arbitrary means between harmonic and arithmetic means and $p>0$. Then

$$
\Phi^{p}(A \sigma B) \Phi^{-p}(A \tau B)+\Phi^{-p}(A \tau B) \Phi^{p}(A \sigma B) \leq 2 \alpha^{p}
$$

where $\alpha=\max \left\{\frac{(M+m)^{2}}{4 M m}, \frac{(M+m)^{2}}{4^{\frac{1}{p}} M m}\right\}$.

Proof. By [5, Lemma 3.5.12] we have that $\|X\| \leq t$ if and only if $\left(\begin{array}{cc}t I & X \\ X^{*} & t I\end{array}\right) \geq 0$, for any $X \in \mathbb{B}(\mathscr{H})$. If $0<p \leq 1$, then $\alpha=\frac{(M+m)^{2}}{4 M m}$. Applying inequality (1.9) and Lemma 2.3 we get

$$
\left\|\Phi^{p}(A \sigma B) \Phi^{-p}(A \tau B)\right\| \leq \alpha^{p} .
$$

Hence

$$
\left(\begin{array}{cc}
\alpha^{p} I & \Phi^{p}(A \sigma B) \Phi^{-p}(A \tau B) \\
\Phi^{-p}(A \tau B) \Phi^{p}(A \sigma B) & \alpha^{p} I
\end{array}\right) \geq 0
$$

and

$$
\left(\begin{array}{cc}
\alpha^{p} I & \Phi^{-p}(A \tau B) \Phi^{p}(A \sigma B) \\
\Phi^{p}(A \sigma B) \Phi^{-p}(A \tau B) & \alpha^{p} I
\end{array}\right) \geq 0
$$

Hence

$$
\left(\begin{array}{cc}
2 \alpha^{p} I & \Phi^{-p}(A \tau B) \Phi^{p}(A \sigma B)+\Phi^{p}(A \sigma B) \Phi^{-p}(A \tau B) \\
\Phi^{p}(A \sigma B) \Phi^{-p}(A \tau B)+\Phi^{-p}(A \tau B) \Phi^{p}(A \sigma B) & 2 \alpha^{p} I
\end{array}\right)
$$

is positive and the desired inequality for $0<p \leq 1$. Using inequality (1.9) with the same argument, we get the desired inequality for $p>1$.

Now, we are ready to present our main result. We need the following lemma, proved in [7]; (see also [2]).

Lemma 2.6. [7] Let $a, b>0$ and $\nu \in[0,1]$. Then

$$
a^{1-\nu} b^{\nu}+r(\sqrt{a}-\sqrt{b})^{2} \leq(1-\nu) a+\nu b,
$$

where $r=\min \{\nu, 1-\nu\}$.

Theorem 2.7. Let $0<m \leq A, B \leq M$, $\Phi$ be a positive unital linear map, $\nu \in[0,1]$ and $p>0$. Then

$$
\Phi^{p}\left(A \nabla_{\nu} B+2 r M m\left(A^{-1} \nabla B^{-1}-A^{-1} \sharp B^{-1}\right)\right) \leq \alpha^{p} \Phi^{p}\left(A \sharp_{\nu} B\right)
$$

and

$$
\Phi^{p}\left(A \nabla_{\nu} B+2 r M m\left(A^{-1} \nabla B^{-1}-A^{-1} \sharp B^{-1}\right)\right) \leq \alpha^{p}\left(\Phi(A) \sharp_{\nu} \Phi(B)\right)^{p},
$$

where $r=\min \{\nu, 1-\nu\}$ and $\alpha=\max \left\{\frac{(M+m)^{2}}{4 M m}, \frac{(M+m)^{2}}{4^{\frac{2}{p}} M m}\right\}$. 
Proof. We prove first the inequalities (2.2) and (2.3) for $0<p \leq 2$. Since $0<m \leq$ $A, B \leq M$ we get that

$$
A+M m A^{-1} \leq M+m \quad \text { and } \quad B+M m B^{-1} \leq M+m .
$$

Therefore, for a positive unital linear map $\Phi$ we have

$$
\Phi(A)+M m \Phi\left(A^{-1}\right) \leq M+m
$$

and

$$
\Phi(B)+M m \Phi\left(B^{-1}\right) \leq M+m .
$$

Obviously we have also the inequalities

$$
\Phi((1-\nu) A)+M m \Phi\left((1-\nu) A^{-1}\right) \leq(1-\nu) M+(1-\nu) m
$$

and

$$
\Phi(\nu B)+M m \Phi\left(\nu B^{-1}\right) \leq \nu M+\nu m
$$

for any $\nu \in[0,1]$. Summing up, we therefore get

$$
\Phi\left(A \nabla_{\nu} B\right)+M m \Phi\left((1-\nu) A^{-1}+\nu B^{-1}\right) \leq M+m .
$$

Moreover, by using the inequality (2.1) and functional calculus for the positive operator $A^{\frac{1}{2}} B^{-1} A^{\frac{1}{2}}$ we have

$$
\left(A^{\frac{1}{2}} B^{-1} A^{\frac{1}{2}}\right)^{\nu}+r\left(A^{\frac{1}{2}} B^{-1} A^{\frac{1}{2}}+1-2\left(A^{\frac{1}{2}} B^{-1} A^{\frac{1}{2}}\right)^{\frac{1}{2}}\right) \leq(1-\nu)+\nu A^{\frac{1}{2}} B^{-1} A^{\frac{1}{2}} .
$$

Multiplying both sides of the above inequality both to the left and to the right by $A^{\frac{-1}{2}}$ we get that

$$
A^{-1} \sharp_{\nu} B^{-1}+2 r\left(A^{-1} \nabla B^{-1}-A^{-1} \sharp B^{-1}\right) \leq(1-\nu) A^{-1}+\nu B^{-1} .
$$


Applying (1.11), (2.4), (2.5) and taking into account the properties of $\Phi$ we have

$$
\begin{aligned}
& \left\|\Phi\left(A \nabla_{\nu} B+2 r M m\left(A^{-1} \nabla B^{-1}-A^{-1} \sharp B^{-1}\right)\right) M m \Phi^{-1}\left(A \sharp_{\nu} B\right)\right\| \\
& \leq \frac{1}{4}\left\|\Phi\left(A \nabla_{\nu} B+2 r M m\left(A^{-1} \nabla B^{-1}-A^{-1} \sharp B^{-1}\right)\right)+M m \Phi^{-1}\left(A \sharp{ }_{\nu} B\right)\right\|^{2}
\end{aligned}
$$

(by Lemma 2.1)

$$
\leq \frac{1}{4}\left\|\Phi\left(A \nabla_{\nu} B+2 r M m\left(A^{-1} \nabla B^{-1}-A^{-1} \sharp B^{-1}\right)\right)+M m \Phi\left(A_{\sharp_{\nu}}^{-B^{-1}}\right)\right\|^{2}
$$

(by inequality (1.11))

$$
\begin{aligned}
& =\frac{1}{4}\left\|\Phi\left(A \nabla_{\nu} B\right)+M m \Phi\left(A^{-1} \sharp_{\nu} B^{-1}+2 r\left(A^{-1} \nabla B^{-1}-A^{-1} \sharp B^{-1}\right)\right)\right\|^{2} \\
& \leq \frac{1}{4}\left\|\Phi\left(A \nabla_{\nu} B\right)+M m \Phi\left((1-\nu) A^{-1}+\nu B^{-1}\right)\right\|^{2} \quad \text { (by inequality (2.5)) } \\
& \left.\leq \frac{1}{4}(M+m)^{2} \quad \text { (by inequality }(2.4)\right) .
\end{aligned}
$$

Therefore

$$
\left\|\Phi\left(A \nabla_{\nu} B+2 r M m\left(A^{-1} \nabla B^{-1}-A^{-1} \sharp B^{-1}\right)\right) \Phi^{-1}(A \sharp \nu B)\right\| \leq \frac{(M+m)^{2}}{4 M m} .
$$

Hence

$$
\Phi^{2}\left(A \nabla_{\nu} B+2 r M m\left(A^{-1} \nabla B^{-1}-A^{-1} \sharp B^{-1}\right)\right) \leq\left(\frac{(M+m)^{2}}{4 M m}\right)^{2} \Phi^{2}\left(A \sharp_{\nu} B\right) .
$$

Since $0<p / 2 \leq 1$, by inequality (1.2) we have

$$
\Phi^{p}\left(A \nabla_{\nu} B+2 r M m\left(A^{-1} \nabla B^{-1}-A^{-1} \sharp B^{-1}\right)\right) \leq\left(\frac{(M+m)^{2}}{4 M m}\right)^{p} \Phi^{p}\left(A \sharp_{\nu} B\right) .
$$

Thus we get the inequality (2.2) for $0<p \leq 2$. We prove now (2.3) for $0<p \leq 2$. Applying Lemma 2.1 and then inequality (2.2) we have

$$
\begin{aligned}
& \left\|\Phi\left(A \nabla_{\nu} B+2 r M m\left(A^{-1} \nabla B^{-1}-A^{-1} \sharp B^{-1}\right)\right) M m\left(\Phi(A) \sharp_{\nu} \Phi(B)\right)^{-1}\right\| \\
& \leq \frac{1}{4}\left\|\Phi\left(A \nabla_{\nu} B+2 r M m\left(A^{-1} \nabla B^{-1}-A^{-1} \sharp B^{-1}\right)\right)+M m\left(\Phi(A) \sharp_{\nu} \Phi(B)\right)^{-1}\right\|^{2} \\
& \quad(\text { by Lemma 2.1) } \\
& \leq \frac{1}{4}\left\|\Phi\left(A \nabla_{\nu} B+2 r M m\left(A^{-1} \nabla B^{-1}-A^{-1} \sharp B^{-1}\right)\right)+M m \Phi^{-1}\left(A \sharp_{\nu} B\right)\right\|^{2} \\
& \leq \frac{1}{4}(M+m)^{2} \quad \text { (by inequality (2.6)). }
\end{aligned}
$$

Hence the inequality $(2.3)$ for $0<p \leq 2$.

Now, we prove the inequalities (2.2) and (2.3) for $p>2$. Then, by Lemma 2.1 and 
2.2 we get

$$
\begin{aligned}
M^{\frac{p}{2}} & m^{\frac{p}{2}}\left\|\Phi^{\frac{p}{2}}\left(A \nabla_{\nu} B+2 r M m\left(A^{-1} \nabla B^{-1}-A^{-1} \sharp B^{-1}\right)\right) \Phi^{\frac{-p}{2}}\left(A \sharp_{\nu} B\right)\right\| \\
& =\left\|\Phi^{\frac{p}{2}}\left(A \nabla_{\nu} B+2 r M m\left(A^{-1} \nabla B^{-1}-A^{-1} \sharp B^{-1}\right)\right) M^{\frac{p}{2}} m^{\frac{p}{2}} \Phi^{\frac{-p}{2}}\left(A \sharp_{\nu} B\right)\right\| \\
& \leq \frac{1}{4}\left\|\Phi^{\frac{p}{2}}\left(A \nabla_{\nu} B+2 r M m\left(A^{-1} \nabla B^{-1}-A^{-1} \sharp B^{-1}\right)\right)+M^{\frac{p}{2}} m^{\frac{p}{2}} \Phi^{\frac{-p}{2}}\left(A \sharp_{\nu} B\right)\right\|^{2} \\
& \leq \frac{1}{4}\left\|\left(\Phi\left(A \nabla_{\nu} B+2 r M m\left(A^{-1} \nabla B^{-1}-A^{-1} \sharp B^{-1}\right)\right)+M m \Phi^{-1}\left(A \sharp_{\nu} B\right)\right)^{\frac{p}{2}}\right\|^{2} \\
& =\frac{1}{4}\left\|\Phi\left(A \nabla_{\nu} B+2 r M m\left(A^{-1} \nabla B^{-1}-A^{-1} \sharp B^{-1}\right)\right)+M m \Phi^{-1}\left(A \sharp_{\nu} B\right)\right\|^{p} \\
& \leq \frac{1}{4}(M+m)^{p} .
\end{aligned}
$$

Hence we get the inequality (2.2) for $p>2$. Further, we have

$$
\begin{aligned}
M^{\frac{p}{2}} & m^{\frac{p}{2}}\left\|\Phi^{\frac{p}{2}}\left(A \nabla_{\nu} B+2 r M m\left(A^{-1} \nabla B^{-1}-A^{-1} \sharp B^{-1}\right)\right)\left(\Phi(A) \sharp_{\nu} \Phi(B)\right)^{\frac{-p}{2}}\right\| \\
& =\left\|\Phi^{\frac{p}{2}}\left(A \nabla_{\nu} B+2 r M m\left(A^{-1} \nabla B^{-1}-A^{-1} \sharp B^{-1}\right)\right) M^{\frac{p}{2}} m^{\frac{p}{2}}\left(\Phi(A) \sharp_{\nu} \Phi(B)\right)^{\frac{-p}{2}}\right\| \\
& \leq \frac{1}{4}\left\|\Phi^{\frac{p}{2}}\left(A \nabla_{\nu} B+2 r M m\left(A^{-1} \nabla B^{-1}-A^{-1} \sharp B^{-1}\right)\right)+M^{\frac{p}{2}} m^{\frac{p}{2}}\left(\Phi(A) \sharp_{\nu} \Phi(B)\right)^{\frac{-p}{2}}\right\|^{2} \\
& \leq \frac{1}{4}\left\|\left(\Phi\left(A \nabla_{\nu} B+2 r M m\left(A^{-1} \nabla B^{-1}-A^{-1} \sharp B^{-1}\right)\right)+M m\left(\Phi(A) \sharp_{\nu} \Phi(B)\right)^{-1}\right)^{\frac{p}{2}}\right\|^{2} \\
& =\frac{1}{4}\left\|\Phi\left(A \nabla_{\nu} B+2 r M m\left(A^{-1} \nabla B^{-1}-A^{-1} \sharp B^{-1}\right)\right)+M m\left(\Phi(A) \sharp_{\nu} \Phi(B)\right)^{-1}\right\|^{p} \\
& \leq \frac{1}{4}\left\|\Phi\left(A \nabla_{\nu} B+2 r M m\left(A^{-1} \nabla B^{-1}-A^{-1} \sharp B^{-1}\right)\right)+M m \Phi^{-1}\left(A \sharp{ }_{\nu} B\right)\right\|^{p} \\
& \leq \frac{1}{4}(M+m)^{p} .
\end{aligned}
$$

Thus we get the inequality (2.3) for $p>2$ and this completes the proof of the theorem.

Remark 2.8. Let $0<m \leq A, B \leq M$, $\Phi$ be a positive unital linear map. If $0<p \leq 1$, then, obviously,

$$
\Phi^{p}\left(A \nabla_{\nu} B\right) \leq\left(\Phi\left(A \nabla_{\nu} B\right)+2 r M m \Phi\left(A^{-1} \nabla B^{-1}-A^{-1} \sharp B^{-1}\right)\right)^{p} .
$$

Hence the inequality (2.7) shows that Theorem 2.7 is a refinement of inequalities (1.5) and (1.6) for $0<p \leq 1$. 
We also have

$$
\Phi^{p}\left(A \nabla_{\nu} B\right) \leq \Phi^{p}\left(A \nabla_{\nu} B\right)+(2 r M m)^{p} \Phi^{p}\left(A^{-1} \nabla B^{-1}-A^{-1} \sharp B^{-1}\right),
$$

where $p \geq 1, \nu \in[0,1]$ and $r=\min \{\nu, 1-\nu\}$.

Hence

$$
\begin{aligned}
\left\|\Phi^{p}\left(A \nabla_{\nu} B\right)\right\| & \leq\left\|\Phi^{p}\left(A \nabla_{\nu} B\right)+(2 r M m)^{p} \Phi^{p}\left(A^{-1} \nabla B^{-1}-A^{-1} \sharp B^{-1}\right)\right\| \\
& \leq\left\|\Phi^{p}\left(A \nabla_{\nu} B+2 r M m A^{-1} \nabla B^{-1}-A^{-1} \sharp B^{-1}\right)\right\| \quad \text { (by Lemma 2.2). }
\end{aligned}
$$

Therefore, Theorem 2.7 is a refinement of the inequalities, (1.7) and (1.8) for the operator norm and $p \geq 2$.

The following examples show that inequality (2.2) is a refinement of (1.5) and $(1.7)$.

Example 2.9. If $A=\left(\begin{array}{cc}1.75 & 0.433 \\ 0.433 & 1.25\end{array}\right), B=\left(\begin{array}{cc}2.5 & 0.5 \\ 0.5 & 2.5\end{array}\right), \Phi(X)=\frac{1}{2} \operatorname{tr}(\mathrm{X})(\mathrm{X} \in$ $\left.\mathbb{M}_{2}\right), m=1, M=3, \nu=\frac{1}{2}$ and $p=3$, then $A \nabla_{\nu} B=\left(\begin{array}{ll}2.1250 & 0.4665 \\ 0.4665 & 1.8750\end{array}\right)$ and $A \nabla_{\nu} B+2 r M m\left(A^{-1} \nabla B^{-1}-A^{-1} \sharp B^{-1}\right)=\left(\begin{array}{ll}2.1601 & 0.4260 \\ 0.4260 & 2.0016\end{array}\right)$. Hence

$$
\Phi^{3}\left(A \nabla_{\nu} B+2 r M m\left(A^{-1} \nabla B^{-1}-A^{-1} \sharp B^{-1}\right)\right)-\Phi^{3}\left(A \nabla_{\nu} B\right)=9.0095-8=1.0095>0 .
$$

Example 2.10. Let $\Phi(X)=T^{*} X T\left(X \in \mathbb{M}_{2}\right)$, where $T=\left(\begin{array}{cc}\frac{\sqrt{2}}{2} & \frac{\sqrt{2}}{2} \\ -\frac{\sqrt{2}}{2} & \frac{\sqrt{2}}{2}\end{array}\right)$. If $A=\left(\begin{array}{cc}5 & -2 \\ -2 & 5\end{array}\right), B=\left(\begin{array}{cc}4.75 & 0.433 \\ 0.433 & 4.25\end{array}\right), m=3, M=7, \nu=\frac{1}{2}$ and $p=\frac{5}{3}$, then $A \nabla_{\nu} B=\left(\begin{array}{cc}4.8750 & -0.7835 \\ -0.7835 & 4.6250\end{array}\right)$ and $A \nabla_{\nu} B+2 r M m\left(A^{-1} \nabla B^{-1}-A^{-1} \sharp B^{-1}\right)=$ $\left(\begin{array}{cc}5.0283 & -0.7730 \\ -0.7730 & 4.7909\end{array}\right)$. Hence

$$
\Phi^{\frac{5}{3}}\left(A \nabla_{\nu} B+2 r M m\left(A^{-1} \nabla B^{-1}-A^{-1} \sharp B^{-1}\right)\right)-\Phi^{\frac{5}{3}}\left(A \nabla_{\nu} B\right)=\left(\begin{array}{cc}
0.7838 & -1.0172 \\
-1.0172 & 0.7199
\end{array}\right)>0 .
$$

Corollary 2.11. Let $0<m \leq A, B \leq M$ and $\Phi$ be a positive unital linear map. Then

$$
\Phi^{p}\left(\frac{A+B}{2}+M m\left(A^{-1} \nabla B^{-1}-A^{-1} \sharp B^{-1}\right)\right) \leq \alpha^{p} \Phi^{p}(A \sharp B)
$$


and

$$
\Phi^{p}\left(\frac{A+B}{2}+M m\left(A^{-1} \nabla B^{-1}-A^{-1} \sharp B^{-1}\right)\right) \leq \alpha^{p}(\Phi(A) \sharp \Phi(B))^{p} .
$$

Proof. Take $r=\nu=\frac{1}{2}$ in Theorem 2.7.

If the positive unital linear map $\Phi(A)=A(A \in \mathbb{B}(\mathscr{H}))$, then we get from Theorem 2.7 the following reverse AM-GM inequalities, which improve the reversed AM-GM inequality (1.1).

Corollary 2.12. Let $0<m \leq A, B \leq M$. Then, the inequalities

$$
\left(\frac{A+B}{2}+M m\left(A^{-1} \nabla B^{-1}-A^{-1} \sharp B^{-1}\right)\right)^{p} \leq\left(\frac{(M+m)^{2}}{4 M m}\right)^{p}(A \sharp B)^{p} \quad(0<p \leq 2)
$$

and

$$
\left(\frac{A+B}{2}+M m\left(A^{-1} \nabla B^{-1}-A^{-1} \sharp B^{-1}\right)\right)^{p} \leq\left(\frac{(M+m)^{2}}{4^{2 / p} M m}\right)^{p} \quad(A \sharp B)^{p} \quad(p>2) .
$$

hold.

The operator Pólya-Szegö inequality states that

$$
\Phi(A) \sharp \Phi(B) \leq \frac{M+m}{2 \sqrt{m M}} \Phi(A \sharp B) .
$$

where $0<m_{1}^{2} \leq A \leq M_{1}^{2}, 0<m_{2}^{2} \leq B \leq M_{2}^{2}, m=\frac{m_{2}}{M_{1}}$ and $M=\frac{M_{1}}{m_{2}}$. Also the operator Kantorovich inequality says that

$$
\Phi(A) \sharp \Phi\left(A^{-1}\right) \leq \frac{M^{2}+m^{2}}{2 m M},
$$

where $0<m_{1}^{2} \leq A \leq M_{1}^{2}, 0<m_{2}^{2} \leq B \leq M_{2}^{2}, m=\frac{m_{2}}{M_{1}}, M=\frac{M_{1}}{m_{2}}$; see [6].

In the following result we show some refinements of (2.8) and (2.9).

Theorem 2.13. Let $\Phi$ be a unital positive linear map, $0<m_{1}^{2} \leq A \leq M_{1}^{2}, 0<$ $m_{2}^{2} \leq B \leq M_{2}^{2}, m=\frac{m_{2}}{M_{1}}, M=\frac{M_{1}}{m_{2}}$.

$$
\Phi(A) \sharp \Phi(B)+\frac{1}{2}\left(\sqrt{M m} \Phi(A)+\frac{1}{\sqrt{M m}} \Phi(B)-2(\Phi(A) \sharp \Phi(B))\right) \leq \frac{M+m}{2 \sqrt{m M}} \Phi(A \sharp B) .
$$

In particular, if $B=A^{-1}$, then

$$
\Phi(A) \sharp \Phi\left(A^{-1}\right)+\frac{1}{2}\left(M m \Phi(A)+\frac{1}{M m} \Phi\left(A^{-1}\right)-2\left(\Phi(A) \sharp \Phi\left(A^{-1}\right)\right)\right) \leq \frac{M^{2}+m^{2}}{2 m M} .
$$

Proof. If $0<m_{1}^{2} \leq A \leq M_{1}^{2}$ and $0<m_{2}^{2} \leq B \leq M_{2}^{2}$, then

$$
m^{2}=\frac{m_{2}^{2}}{M_{1}^{2}} \leq A^{\frac{-1}{2}} B A^{\frac{-1}{2}} \leq \frac{M_{1}^{2}}{m_{2}^{2}}=M^{2}
$$


whence

$$
\left(M-\left(A^{\frac{-1}{2}} B A^{\frac{-1}{2}}\right)^{\frac{1}{2}}\right)\left(\left(A^{\frac{-1}{2}} B A^{\frac{-1}{2}}\right)^{\frac{1}{2}}-m\right) \geq 0 .
$$

Hence

$$
M m A+B \leq(M+m) A \sharp B,
$$

whence

$$
M m \Phi(A)+\Phi(B) \leq(M+m) \Phi(A \sharp B) .
$$

Using lemma 2.1 for the operators $\operatorname{Mm} \Phi(A), \Phi(B)$ and $\nu=\frac{1}{2}$ we get

$$
\sqrt{M m}(\Phi(A) \sharp \Phi(B))+\frac{1}{2}(M m \Phi(A)+\Phi(B)-2 \sqrt{M m}(\Phi(A) \sharp \Phi(B))) \leq \frac{1}{2}(M m \Phi(A)+\Phi(B)) .
$$

Applying inequalities (2.11) and (2.12) we get the first inequality. In particular, if we consider $m_{1}^{2}=m^{2} \leq A \leq M^{2}=M_{1}^{2}$, then by putting $m_{2}^{2}=\frac{1}{M^{2}} \leq A^{-1} \leq \frac{1}{m^{2}}=M_{2}^{2}$ in (2.10) we reach the desired inequality.

If we take $\Phi$ in (2.10) to be the positive linear map defined on the diagonal blocks of operators by $\Phi\left(\operatorname{diag}\left(A_{1}, \cdots, A_{n}\right)\right)=\frac{1}{n} \sum_{j=1}^{n} A_{j}$, then we get the following refinements of a reversed Cauchy-Schwarz operator inequality.

Corollary 2.14. Let $0<m_{1}^{2} \leq A_{j} \leq M_{1}^{2}, 0<m_{2}^{2} \leq B_{j} \leq M_{2}^{2}(1 \leq j \leq n)$, $m=\frac{m_{2}}{M_{1}}, M=\frac{M_{1}}{m_{2}}$. Then

$$
\begin{array}{r}
\left(\sum_{j=1}^{n} A_{j} \sharp \sum_{j=1}^{n} B_{j}\right)+\frac{1}{2}\left(\sqrt{M m} \sum_{j=1}^{n} A_{j} \frac{1}{\sqrt{M m}} \sum_{j=1}^{n} B_{j}-2\left(\sum_{j=1}^{n} A_{j} \sharp \sum_{j=1}^{n} B_{j}\right)\right) \\
\leq \frac{M+m}{2 \sqrt{m M}}\left(\sum_{j=1}^{n} A_{j} \sharp B_{j}\right) .
\end{array}
$$

Proposition 2.15. Let $0<m \leq A \leq M$ and $x \in \mathscr{H}$. Then

$$
\langle A x, x\rangle^{\frac{1}{2}}\left\langle A^{-1} x, x\right\rangle^{\frac{1}{2}}+\frac{1}{2}\left(\sqrt[4]{M m}\langle A x, x\rangle^{\frac{1}{2}}-\frac{1}{\sqrt[4]{M m}}\left\langle A^{-1} x, x\right\rangle^{\frac{1}{2}}\right)^{2} \leq \frac{M+m}{2 \sqrt{M m}}\langle x, x\rangle^{2} .
$$




\section{REFERENCES}

1. R. Bhatia, Positive Definite Matrices, Princeton University Press, Princeton, 2007.

2. M. Bakherad and M.S. Moslehian, Reverses and variations of Heinz inequality, Linear Multilinear Algebra, http://dx.doi.org/10.1080/03081087.2014.880433.

3. R. Bhatia and F. Kittaneh, Notes on matrix arithmetic-geometric mean inequalities, Linear Algebra Appl. 308 (2000), no. 1-3, 203-211.

4. D.T. Hoa, D.T.H. Binh and H.M. Toan, On some inequalities with matrix means, RIMS Kokyuroku, no. 1893, (2014)-05, 67-71, Kyoto University.

5. R.A. Horn and C.R. Johnson, Topics in Matrix Analysis, Cambridge University Press, 1991.

6. R. Kaur, M. Singh, J.S. Aujla and M.S. Moslehian, A general double inequality related to operator means and positive linear maps, Linear Algebra Appl 437 (2012), no. 3, 1016-1024.

7. F. Kittaneh and Y. Manasrah, Improved Young and Heinz inequalities for matrices, J. Math. Anal. Appl. 36 (2010), 262-269.

8. F. Kubo and T. Ando, Means of positive linear operators, Math. Ann. 246 (1980), 205-224.

9. M. Tominaga, Specht's ratio in the Young inequality, Sci. Math. Japan. 55 (2002), no. 3, $583-588$.

10. M. Lin, Squaring a reverse AM-GM inequality, Studia Math. 215 (2013), no. 2, 187-194.

11. M. Lin, On an operator Kantorovich inequality for positive linear maps, J. Math. Anal. Appl. 402 (2013), 127-132.

12. X. Fu and D.T. Hoa, On some inequalities with matrix means, Linear Multilinear Algebra,(2015) http://dx.doi.org/10.1080/03081087.2015.1010472.

13. X. Fu and C. He, Some operator inequalities for positive linear maps, Linear Multilinear Algebra, 63 (2015), no. 3, 571-577.

Department of Mathematics, Faculty of Mathematics, University of Sistan and Baluchestan, P.O. Box 98135-674, Zahedan, Iran.

E-mail address: mojtaba.bakherad@yahoo.com; bakherad@member.ams.org 